\title{
On the use of NURBS plasticity for geomaterials
}

\author{
W.M. Coombs \\ Department of Engineering \\ Durham University, Durham, $U K$ \\ Y. Ghaffari Motlagh \\ School of Chemical and Process Engineering \\ University of Leeds, Leeds, UK.
}

\begin{abstract}
There are a huge number of plasticity models available in the literature to represent the behaviour of geomaterials and the majority include the concept of a yield surface which bounds the allowable stress in the material. However, each surface is distinct and requires a specific equation describing the shape of the surface to be formulated in each case. Recently Coombs et al. (2016) proposed a non-uniform rational basis spline (NURBS) plasticity framework where any isotropic yield surface can be represented and integrated using the same numerical algorithm. However, the NURBS plasticity framework is currently restricted the to case of associated plastic flow which will lead to an overly dilative response for most geomaterials. This paper extends the approach to include a non-associated plastic flow rule whilst guaranteeing the recovery of associated plastic flow if required. The algorithm's performance is demonstrated at both material point (stress-strain) and boundary value problem levels.
\end{abstract}

\section{INTRODUCTION}

Numerical analysis of engineering problems rely on robust and efficient constitutive models that provide the link between stress and strain in the material under consideration. Within this, conventional plasticity models use the concept of a yield surface to distinguish between elastic (inside the surface) and elastoplastic (on the surface) behaviour. Depending on the material analysed the shape of this surface will change and this impacts on the stress integration algorithm ${ }^{1}$ (which requires changes in the numerics) for each implemented yield surface. The non-uniform rational basis spline (NURBS) plasticity framework was first proposed by Coombs et al. (2016) and extended to include linear isotropic hardening by Coombs and Ghaffari Motlagh (2017). The key idea of the framework is to represent the yield surface of an isotropic plasticity model using a NURBS surface. This allows different yield criteria to be included within the same numerical algorithm by only changing information associated with the NURBS surface (control point positions, spline order, etc.).

\footnotetext{
${ }^{1}$ A stress integration algorithm is required to convert the constitutive equations, which are typically developed in rate form, into an incremental relationship that can be used within a boundary value solver (finite elements, for example).
}

This paper adopts the NURBS plasticity framework and applies it to yield surfaces typically used for the behaviour of geomaterials, such as Mohr-Coulomb and Drucker-Prager. However, it is well known that associated plastic flow frictional yield surfaces overestimate dilative behaviour. Therefore this paper extends the framework to include non-associated plastic flow whilst maintaining that all information associated with the yielding of the material is held at control points.

The layout of the paper is as follows. After this introduction, Section 2 describes the NURBS plasticity framework and its extension to include nonassociated flow. Section 3 discusses the implementation of the model and Section 4 presents a series of material point and boundary value simulations to demonstrate the capabilities of the non-associated plastic flow NURBS framework. Finally, conclusions are drawn in Section 5.

The majority of this paper is presented in terms of principal stresses with a tension positive notation and the conventional ordering of the principal stresses

$\sigma_{1} \geq \sigma_{2} \geq \sigma_{3}$

which restricts the principal stress state to a single sextant of stress space. Adopting a principal stress notation is common in other isotropic plasticity mod- 
els, for example see the work of Coombs and Crouch (2011a), Coombs et al. (2010), Coombs and Crouch (2011b) and Clausen et al. (2007) amongst others, and does not change the generality of the algorithm (it is suitable for 1D, 2D and 3D analysis). The principal values are simply mapped back to conventional sixcomponent stress space at the end of the constitutive algorithm.

\section{NURBS PLASTICITY}

The following section outlines the NURBS plasticity framework and extends the approach of Coombs et al. (2016) to include non-associated plastic flow.

A general NURBS surface can be expressed as

$S_{k}(\xi, \eta)=\sum_{i=0}^{n} \sum_{j=0}^{m} R_{i, j}(\xi, \eta)\left(C_{k}\right)_{i, j}$

where $k$ is the physical index, $C_{k}$ are the control point positions and $n$ and $m$ are the number of control points in the $\xi$ and $\eta$ directions ${ }^{2}$. The NURBS basis functions, $R_{i, j}$, are given by

$$
R_{i, j}(\xi, \eta)=\frac{N_{i, p}(\xi) N_{j, q}(\eta) w_{i, j}}{\sum_{k=0}^{n} \sum_{l=0}^{m} N_{k, p}(\xi) N_{l, q}(\eta) w_{k, l}}
$$

where $N_{i, p}$ and $N_{j, q}$ are the $p^{t h}$ and $q^{t h}$-degree Bspline basis functions, $\xi$ and $\eta$ are the local positions within the Knot vectors and $w_{i, j}$ are the weights associated with the control points.

\subsection{Yield surfaces \& associated plastic flow}

Within the framework of NURBS plasticity (Coombs et al. 2016), the yield surface can be expressed as

$f=\left(\sigma_{i}-S_{i}(\xi, \eta)\right)\left(S,_{\sigma}\right)_{i}=0$,

where $\sigma_{i}$ is the principal stress state and $\left(S,_{\sigma}\right)_{i}$ is the partial derivative of (1) with respect to stress which is the same as the outward normal to the surface. This can be obtained through the cross product of the two local derivatives

$$
\left(S,_{\sigma}\right)_{i}=\left(S,_{\eta} \times S, \xi\right)_{i}=\epsilon_{i j k}\left(S,_{\eta}\right)_{j}\left(S,_{\xi}\right)_{k},
$$

where $\epsilon_{i j k}$ is the Levi-Civita tensor ${ }^{3}$.

In associated flow plasticity theory the plastic strains evolve according to

$\dot{\varepsilon}_{i}^{\mathrm{p}}=\dot{\gamma}\left(S,{ }_{\sigma}\right)_{i}$,

\footnotetext{
${ }^{2}$ Note that the total number of control points used to define the surface is $n \times m$.

${ }^{3} \epsilon_{i j k}=0$ if $i=j, j=k$ or $k=i, \epsilon_{i j k}=1$ for even permutations of $i, j$ and $k$ and $\epsilon_{i j k}=-1$ for odd permutations of $i, j$ and $k$.
}

where $\dot{\gamma}$ is the scalar plastic multiplier (or consistency parameter). This plastic multiplier must satisfy the Kuhn-Tucker-Karush consistency conditions

$f\left(\sigma_{i}\right) \leq 0, \quad \dot{\gamma} \geq 0 \quad$ and $\quad f\left(\sigma_{i}\right) \dot{\gamma}=0$.

These conditions enforce that the material must either be on the yield surface undergoing elasto-plastic deformation ( $f=0$ and $\dot{\gamma} \geq 0$ ) or inside the yield surface with purely elastic behaviour $(f \leq 0$ and $\dot{\gamma}=0)$.

Figure 1 shows a spherical NURBS surface and associated control points (shown by the red points). Different surfaces can be obtained by moving the positions of the control points and/or modifying the basis functions, $R_{i, j}$.

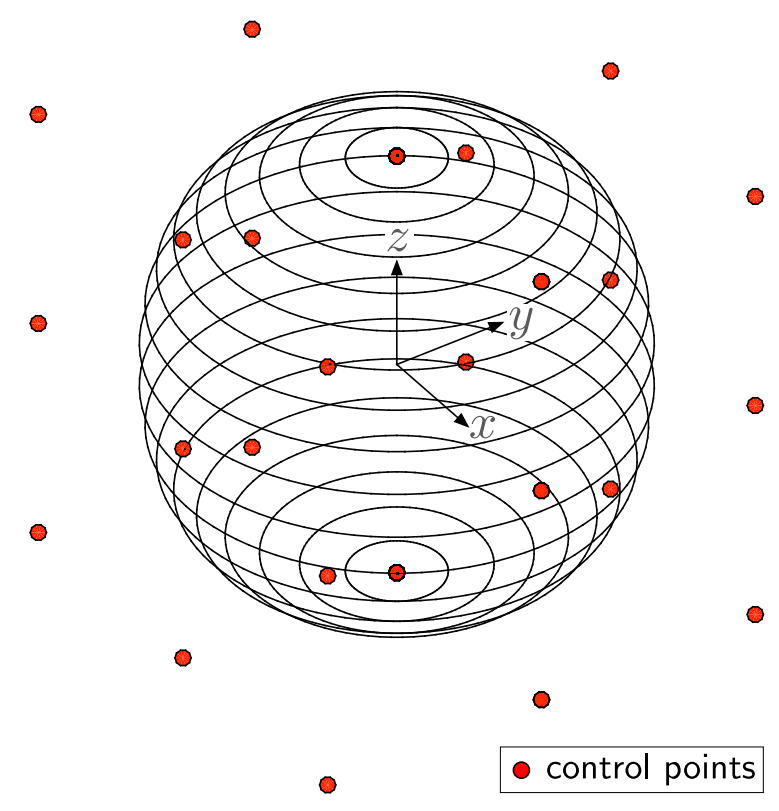

Figure 1: Spherical NURBS surface and control point net where the control points are shown by the red-shaded circles.

\subsection{Non-associated plastic flow}

In the case of non-associated flow the evolution of plastic strains is decoupled from the spatial gradient of the yield envelope and the plastic strains evolve according to

$\dot{\varepsilon}_{i}^{\mathrm{p}}=\dot{\gamma}\left(g,{ }_{\sigma}\right)_{i}$,

where $\left(g,_{\sigma}\right)_{i}$ is the gradient of the plastic potential surface.

In this NURBS plasticity approach the gradient of the plastic potential surface is given by

$\left(g,_{\sigma}\right)_{i}=\left(S^{g}{ }_{\eta} \times S^{g}{ }_{\xi}\right)_{i}=\epsilon_{i j k}\left(S^{g}{ }_{\eta}\right)_{j}\left(S^{g}{ }_{\xi}\right)_{k}$,

where $S^{g}$ is the plastic potential surface

$S_{k}^{g}(\xi, \eta)=\sum_{i=0}^{n} \sum_{j=0}^{m} R_{i, j}^{g}(\xi, \eta)\left(G_{k}\right)_{i, j}$ 
and $G_{k}$ are control point coordinates that control the shape of the surface. The NURBS basis functions $R_{i, j}^{g}(\xi, \eta)$ are calculated in the same way as (2). Note that it is not necessary to have the same basis functions from the direction of plastic flow as used to describe the geometry of the yield surface however it is assumed that a single set of control points control the form of $S_{k}$ and $S_{k}^{g}$.

Previous attempts to extend the NURBS plasticity framework to include non-associated flow have directly specified the flow direction at the control points rather than specifying the geometry of a plastic potential surface (see Coombs (2017)). However, due to the presence of the cross product in (4) it is not possible to specify the flow direction at the control points and guarantee the recovery of associated flow. With this approach it is only possible to recover associated flow over the entire yield surface if there is no coupling between the local knot coordinates $(\xi, \eta)$; the von Mises yield surface is one example. In this paper we adopt a more conventional approach, in terms of plasticity theory, and specify the geometry of the plastic potential surface using control points. In the case of associated plastic flow the plastic potential control point positions coincide with those used to define the geometry of the yield surface.

\subsection{Stress integration}

A robust and efficient stress integration routine is essential if a constitutive model is to be used to analyse engineering problems. These routines convert the rate relationships presented in the previous section into an incremental algorithm that, given the previous stress state and a strain increment, will provide an updated stress state.

In this work we use an implicit elastic predictor, plastic corrector scheme (Wilkins 1964), where the elastic trial stress is given by

$\sigma_{i}^{t}=\sigma_{i}^{n}+\Delta \sigma_{i}$

The stress increment and the previous convergence stress state are given by

$\Delta \sigma_{i}=D_{i j}^{\mathrm{e}} \Delta \varepsilon_{j} \quad$ and $\quad \sigma_{i}^{n}=D_{i j}^{\mathrm{e}}\left(\varepsilon_{n}^{\mathrm{e}}\right)_{j}$.

$\left(\varepsilon_{n}^{\mathrm{e}}\right)_{j}$ is the elastic strain state from the previous load (or time) step in the global solution algorithm, $\Delta \varepsilon_{i}$ is the strain increment associated with the global boundary value displacement and $D_{i j}^{\mathrm{e}}$ contains the principal components of the linear elastic stiffness matrix.

If the trial elastic stress state exceeds the yield envelope $(f>0)$ then it must be corrected back onto the yield surface using a plastic stress increment, that is

$\sigma_{i}^{r}=\sigma_{i}^{t}-\Delta \sigma_{i}^{\mathrm{p}}, \quad$ where $\quad \Delta \sigma_{i}^{\mathrm{p}}=D_{i j}^{\mathrm{e}} \Delta \varepsilon_{j}^{\mathrm{p}}$.

$\sigma_{i}^{r}$ is the returned stress state on the yield surface and $\Delta \epsilon_{j}^{\mathrm{p}}$ is the plastic strain increment obtained from the incremental form of (7). Once this correction has been applied the updated elastic strain can be obtained from

$$
\left(\varepsilon_{n+1}^{\mathrm{e}}\right)_{i}=\left(\varepsilon_{n}^{\mathrm{e}}\right)_{i}+\Delta \varepsilon_{i}-\Delta \varepsilon_{i}^{\mathrm{p}} .
$$

\section{IMPLEMENTATION}

Consistent with the perfect plasticity implementation of Coombs et al. (2016), here we use a coarse initial subdivision algorithm to provide the initial starting point for a backward Euler (bE) implicit stress integration process. This is to provide an initial estimate for the local positions within the Knot vectors, $\xi$ and $\eta$ in (3) that act as the primary unknowns in the implicit bE closest point projection (CPP) problem. However, despite this process being referred to as a CPP, the return stress is not generally the closest point geometrically in standard stress space.

In this paper we make use of energy-mapped space (Crouch et al. 2009) to convert this CPP minimisation into a problem of finding the point on the yield envelope that the normal to the plastic potential surface passes through when intersecting with a trial point outside of the surface. Once the closest point solution in energy-mapped stress space has been found, the solution can be transformed back to conventional stress space. For a NURBS yield surface we only need to map the control point coordinates for both the yield and plastic potential surfaces into energymapped space, the rest of the NURBS information remains unchanged.

As with the algorithm for associated flow perfect plasticity, see Coombs et al. (2016), the stress return path for bE procedure described in this paper starts and remains in the yield envelope and thereby satisfies the consistency conditions not only at the final state but also during the stress updating algorithm. This removes an issue associated with bE stress integration algorithms where they can become trapped in a local minimum, or converge to a spurious auxiliary surface, outside of the true yield surface. Introducing non-associated flow does not increase the number of unknowns in the stress integration algorithm; the key unknowns are the local coordinates on the NURBS surface, $(\xi, \eta)$. The adopted implicit stress integration algorithm also allows the formulation of the algorithmic consistent tangent (Simo and Taylor 1985) which ensures optimum convergence of the global equilibrium equations when implemented within an implicit boundary value solver.

\section{NUMERICAL EXAMPLES}

This section presents a series of numerical examples to demonstrate the performance of the non-associated flow NURBS plasticity formulation.

All of the analyses use a Drucker-Prager (D-P) yield surface (Drucker and Prager 1952) which can 
be expressed as

$f=\rho+\beta\left(\zeta-\zeta_{a}\right)=0$,

where the deviatoric stress is $\rho=\sqrt{2 J_{2}}$ with $J_{2}=$ $\frac{1}{2} \operatorname{tr}\left(s_{i j} s_{j k}\right), s_{i j}=\sigma_{i j}-\frac{1}{3} \sigma_{k k} \delta_{i j}$ and $\delta_{i j}$ the Kronecker delta tensor. $\zeta=\sigma_{i i} / \sqrt{3}$ is the hydrostatic stress, $\beta=\tan (\phi)$ is the opening angle of the cone, $\zeta_{a}=$ $c \sqrt{3} \cot (\phi)$ is the location of the cone's tensile apex, $\phi$ is the friction angle and $c$ the cohesion. The tensile apex of the yield surface poses an issue for the stress return algorithm presented in this paper as the derivatives of the NURBS surface are undefined at this point. Here we follow the same approach as Coombs and Ghaffari Motlagh (2017) and locally round the apex, as shown in Figure 2 with $\zeta_{a}=0$. Both the original (fine lines) and rounded (thick lines) surfaces are shown in principal stress space. The plastic potential surface is similar to (14) but with $\beta$ replaced with $\beta_{g}=\tan (\psi)$ where $\psi \in[0, \phi]$ is the dilation angle. For associated plastic flow $\psi=\phi$.

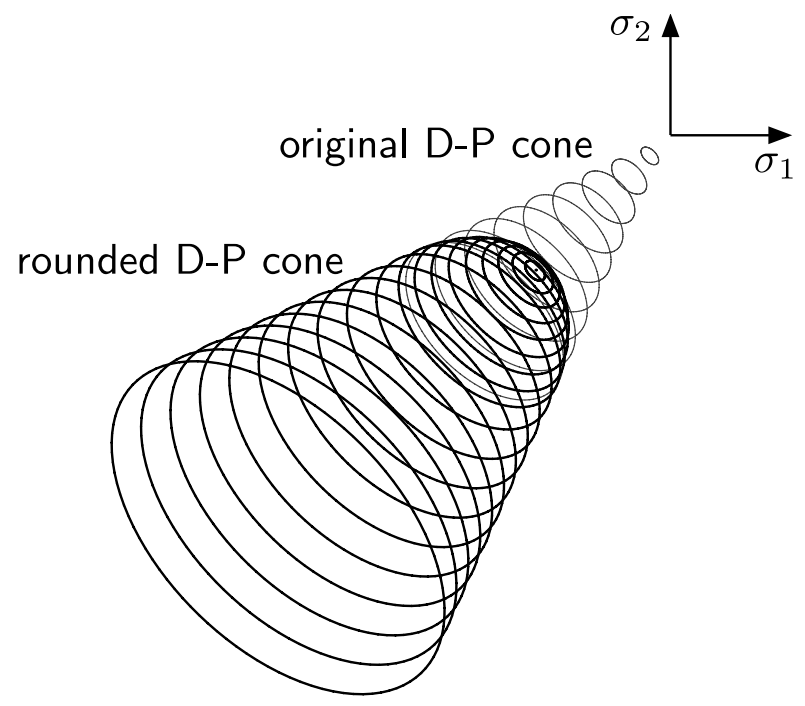

Figure 2: Drucker-Prager yield surface with (exaggerated) apex rounding (reproduced from Coombs and Ghaffari Motlagh (2017)).

Within the framework of NURBS plasticity, the yield envelope is defined in a single sextant of stress space using a bi-quadratic NURBS surface with 15 control points ( 3 in the deviatoric direction by 5 in the hydrostatic direction). All of the examples presented in this section use a friction angle of $\phi=\pi / 9\left(20^{\circ}\right.$ degrees).

\subsection{Material point investigations}

This section analyses the errors associated with the implicit stress integration algorithm. The material had a Young's modulus of $100 \mathrm{kPa}$ and a Poisson's ratio of 0.2 . The cohesion was set to $0.49 \mathrm{kPa}$ and the final $0.1 \mathrm{kPa}$ of the yield surface apex was rounded ${ }^{4}$. The

\footnotetext{
${ }^{4}$ Note that in all cases associated flow was imposed on the rounded part of the yield surface.
}

errors associated with the stress return algorithm were evaluated using dilation angles of $\pi / 18$ and $\pi / 36\left(10^{\circ}\right.$ and $5^{\circ}$ degrees).

The stress state was initially located on the shear meridian in one of the sextants of stress space with a hydrostatic stress of $\zeta=0 \mathrm{kPa}$. This point was then subjected to a stress increment that took the trial stress state outside of the yield envelope. The space of trial states explored was $\rho_{t} / \rho_{n} \in[1,6]$, where the $t$ and $n$ subscripts denote the trial and starting locations.

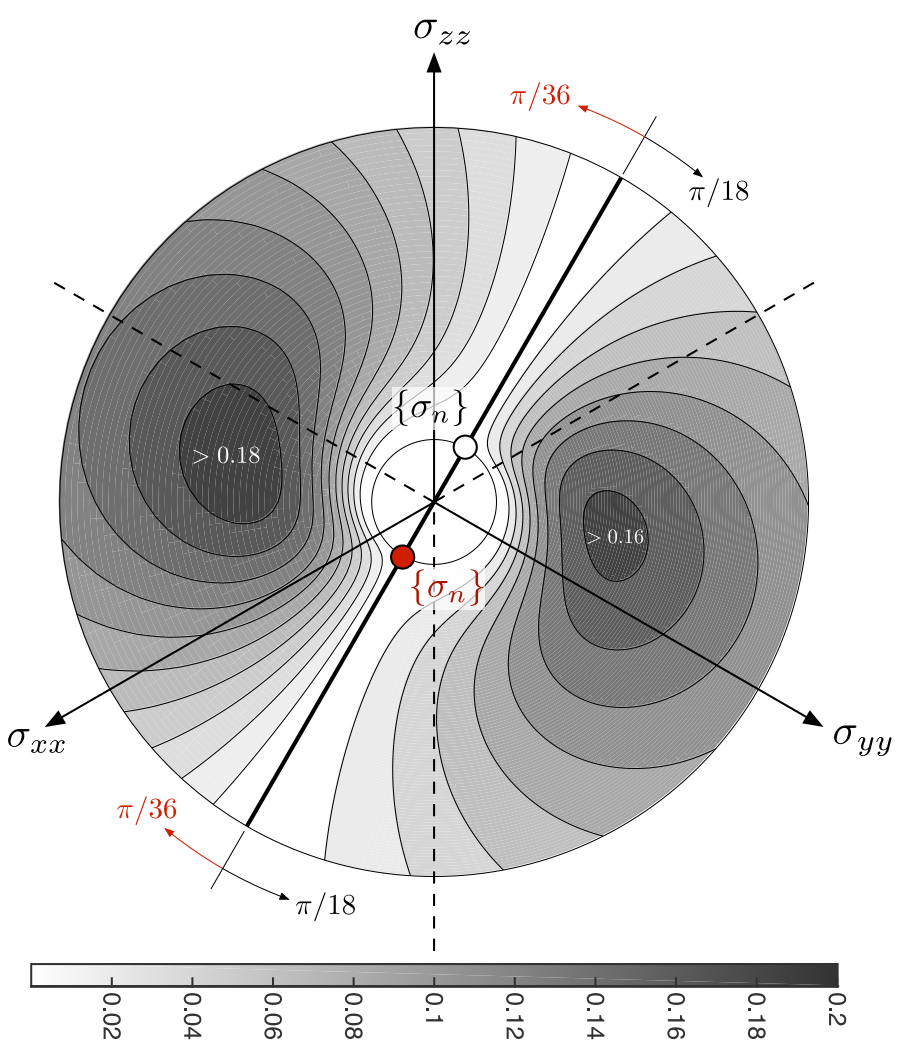

normalised stress return error, $\left\|\left\{\sigma_{\text {NURBS }}\right\}-\left\{\sigma_{e}\right\}\right\| /\left\|\left\{\sigma_{e}\right\}\right\|$

Figure 3: Stress return error analysis for D-P with non-associated flow with $\psi=\pi / 18$ and $\psi=\pi / 36$. The inner circle shows a deviatoric section through the yield surface and the white and red filled circles the starting points for the $\psi=\pi / 18$ and $\psi=\pi / 36$ error analyses, respectively.

The errors associated with the trial state are shown in Figure 3, using the following normalised error measure

error $=\frac{\left\|\left\{\sigma_{\mathrm{NURBS}}\right\}-\left\{\sigma_{e}\right\}\right\|}{\left\|\left\{\sigma_{e}\right\}\right\|}$,

where $\left\{\sigma_{\text {NURBS }}\right\}$ is the stress return location associated with the NURBS model and $\left\{\sigma_{e}\right\}$ is the exact stress return ${ }^{5}$. The errors associated with $\psi=\pi / 18$ and $\psi=\pi / 36$ are shown on the right and left of the thick black line, respectively. The starting point for $\psi=\pi / 18$ is shown by the white-shaded circle whereas the red-shaded circle is the starting point for the $\psi=\pi / 36$ analysis.

\footnotetext{
${ }^{5}$ The exact stress state was approximated by using a conventional implicit stress return algorithm for the D-P model with the stress increment applied in 1000 sub steps.
} 
Although errors of almost $20 \%$ are present in the model, exactly the same level of errors are observed in the D-P yield surface integrated with a conventional implicit stress integration procedure. As expected with any predictor-corrector stress integration algorithm, the error increases as the tangential proportion of the stress increment increases. Errors also increase with increasing non-associativity, with $\psi=\pi / 18$ having a maximum error of $1.66 \times 10^{-1}$ whereas for the $\psi=\pi / 36$ the maximum error was $1.92 \times 10^{-1}$, again this is due to the return path having a larger tangential component relative to the yield surface normal direction.

\subsection{Rigid footing}

The final example is that of a $1 \mathrm{~m}$ wide plane strain rigid smooth footing ${ }^{6}$ displacing into a weightless $10 \mathrm{~m}$ by $5 \mathrm{~m}$ domain with a Young's modulus of $E=$ $1 \times 10^{7} \mathrm{kPa}$ and a Poisson's ratio of $\nu=0.48$. Yielding of the material was governed by a D-P yield envelope with cohesion of $c=490 \mathrm{kPa}$ and a friction angle of $\theta=\pi / 9$ (20 degrees).

The problem was analysed using a mesh comprising 135 eight-noded bi-quadratic quadrilateral elements integrated using reduced four-point quadrature. Due to symmetry only half of the problem was modelled. The mesh is the same as that used by de Souza Neto et al. (2008) (amongst others) and is shown in Figure 4. A vertical displacement of $4 \mathrm{~mm}$ was applied to the footing over 20 equal displacement-controlled loadsteps.

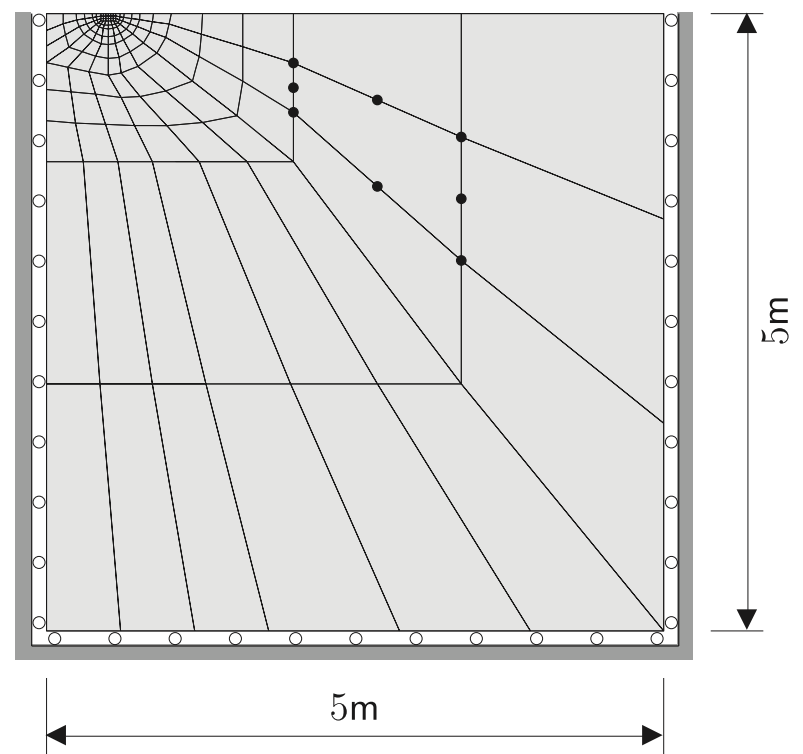

Figure 4: Rigid footing: finite element mesh. The mesh detail around the footing is shown in Figure 5.

The normalised pressure versus displacement response is shown in Figure 5 along with an inset figure showing the mesh detail around the rigid footing. The associate flow (AF) NURBS plasticity re-

\footnotetext{
${ }^{6}$ The term smooth denotes that the nodes underneath the footing are free to displace in horizontal direction.
}

sponse (long dashed line) is compared with the result of de Souza Neto et al. (2008) (discrete points) and that of a conventional backward Euler (bE) closest point projection implementation of the D-P yield surface (thick grey line). Excellent agreement is seen between the three results.

The non-associated flow (NAF) result with $\psi=$ $\pi / 18\left(10^{\circ}\right.$ degrees $)$ is also presented in Figure 5 for both the conventional $\mathrm{bE}$ (thick grey line) and the NURBS (short dashed line) models. As with the associated flow results, excellent agreement is seen between the two models. Combined with the material point investigation presented in the previous section, this verifies the implementation of non-associated flow within the NURBS plasticity framework.

Figure 6 shows the deformed mesh around the footing for associated (top) and non-associated (bottom) plastic flow where the mesh has been shaded by the vertical displacement with dark grey being the maximum downwards displacement. The original mesh is shown by the fine dashed line and the displacements have been exaggerated by $\times 20$. The associated flow case exhibits excessive volumetric dilation in the region of soil adjacent to the footing leading to unrealistic heaving of the ground surface. Reducing the dilation angle from $\pi / 9$ to $\pi / 18$ significantly reduces the heave leading to a more realistic deformed surface profile.

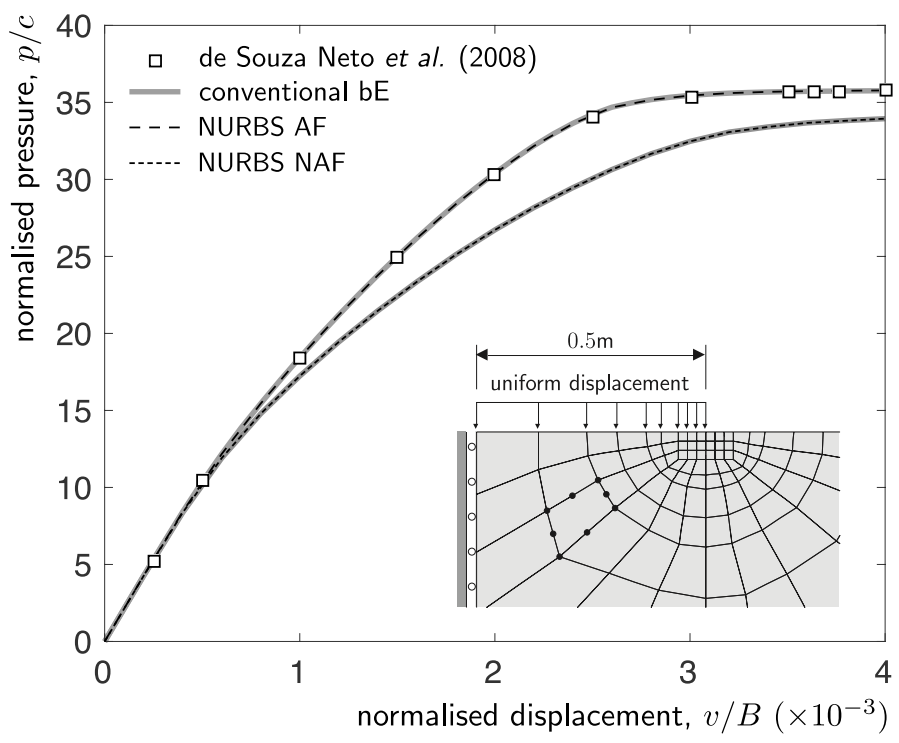

Figure 5: Rigid footing: normalised pressure versus displacement response where $B=1 \mathrm{~m}$ is the footing width.

\section{CONCLUSION}

This paper has applied the NURBS plasticity framework to frictional plasticity models and extended the approach to include non-associated plastic flow. The approach was demonstrated at both a stress-strain and boundary value simulation level and the results validated against published data and conventional constitutive models. Extending the NURBS plasticity approach to include non-associated plastic flow allows 

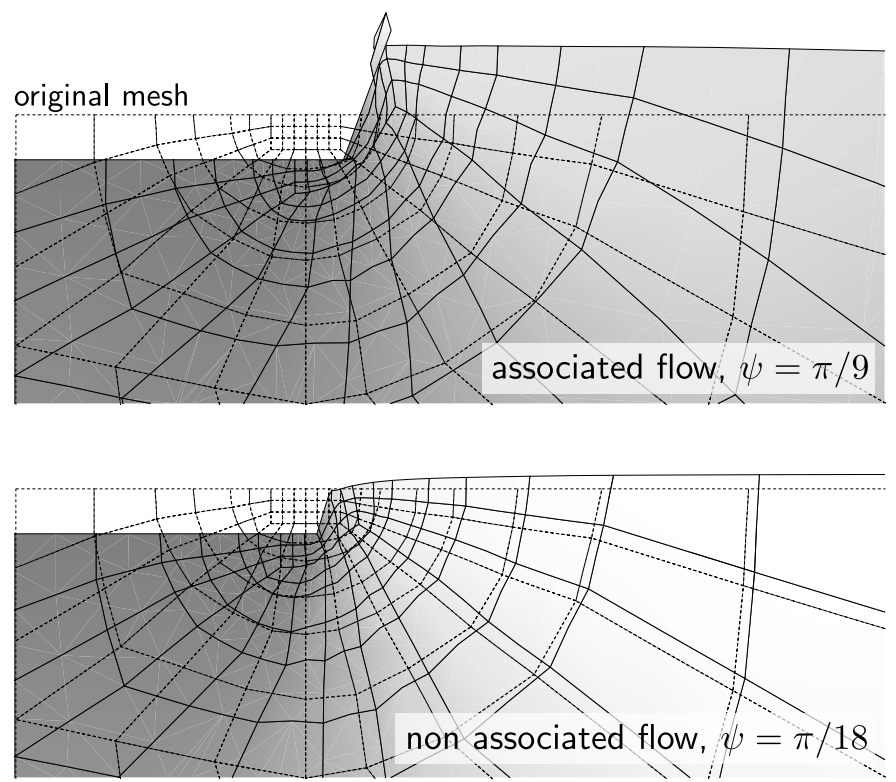

Figure 6: Rigid footing: deformed mesh for associated (top) and non associated flow (bottom) with $\times 20$ displacement magnification.

the framework to model the frictional response of geomaterials in a more realistic way, specifically avoiding the excessive volumetric dilation observed in associated flow plasticity models.

\section{REFERENCES}

Clausen, J., L. Damkilde, \& L. Andersen (2007). An efficient return algorithm for non-associated plasticity with linear yield criteria in principal stress space. Computers \& Structures 85(23-24), 1795-1807.

Coombs, W. M. (2017). Hardening and non-associated flow NURBS plasticity. In E. Oñate, D. Owen, D. Peric, and M. Chiumenti (Eds.), COMPLAS 2017: XIV International Conference on Computational Plasticity: Fundamentals and Applications, Barcelona, Spain, pp. 363-372.

Coombs, W. M. \& R. S. Crouch (2011a). Algorithmic issues for three-invariant hyperplastic critical state models. Comput. Methods Appl. Mech. Engrg. 200(25-28), 2297-2318.

Coombs, W. M. \& R. S. Crouch (2011b). Non-associated Reuleaux plasticity: analytical stress integration and consistent tangent for finite deformation mechanics. Comput. Methods Appl. Mech. Engrg. 200(9-12), 1021-1037.

Coombs, W. M., R. S. Crouch, \& C. E. Augarde (2010). Reuleaux plasticity: analytical backward Euler stress integration and consistent tangent. Comput. Methods Appl. Mech. Engrg. 199(25-28), 1733-1743.

Coombs, W. M. \& Y. Ghaffari Motlagh (2017). NURBS plasticity: yield surface evolution and implicit stress integration for isotropic hardening. Comput. Methods Appl. Mech. Engrg. 324, 204-220.

Coombs, W. M., O. A. Petit, \& Y. Ghaffari Motlagh (2016). NURBS plasticity: Yield surface representation and implicit stress integration for isotropic inelasticity. Comput. Methods Appl. Mech. Engrg. 304, 342 - 358.

Crouch, R. S., H. Askes, \& T. Li (2009). Analytical CPP in energy-mapped stress space: application to a modified Drucker-Prager yield surface. Comput. Methods Appl. Mech. Engrg. 198(5-8), 853-859.

de Souza Neto, E. A., D. Perić, \& D. R. J. Owen (2008). Computational methods for plasticity: Theory and applications. John Wiley \& Sons Ltd.

Drucker, D. \& W. Prager (1952). Soil mechanics and plastic analysis or limit design. Quart. Appl. Math 10(2), 157-164.
Simo, J. C. \& R. L. Taylor (1985). Consistent tangent operators for rate-independent elastoplasticity. Comput. Meth. Appl. Mech. Eng. 48(1), 101-118.

Wilkins, M. L. (1964). Calculation of elastic-plastic flow. Technical report, California Univ Livermore Radiation Lab. 